\title{
A novel method for estimating the focal size of two confocal high-intensity focused ultrasound transducers
}

\author{
Wen-Shiang Chen \\ Department of Physical Medicine and Rehabilitation, National Taiwan University Hospital, Taipei 100, \\ Taiwan and Department of Mechanical Engineering, National Taiwan University, Taipei 106, Taiwan \\ Ping-Mo Ma \\ Department of Mechanical Engineering, National Taiwan University, Taipei 106, Taiwan \\ Hao-Li Liu \\ Department of Electronic Engineering, Tung-Nan Institute of Technology, Taipei, Taiwan \\ Chih-Kuang Yeh ${ }^{\text {a) }}$ \\ Department of Electrical Engineering, Yuan Ze University, Taoyuan 320, Taiwan \\ Min-Shin Chen \\ Department of Mechanical Engineering, National Taiwan University, Taipei 106, Taiwan \\ Chein-Wei Chang \\ Department of Physical Medicine and Rehabilitation, National Taiwan University Hospital, Taipei 100, \\ Taiwan
}

(Received 7 November 2004; revised 4 March 2005; accepted 15 March 2005)

\begin{abstract}
Estimating the focal size and position of a high-intensity focused ultrasound (HIFU) transducer remains a challenge since traditional methods, such as hydrophone scanning or schlieren imaging, cannot tolerate high pressures, are directional, or provide low resolution. The difficulties increase when dealing with the complex beam pattern of a multielement HIFU transducer array, e.g., two transducers facing each other. In the present study we show a novel approach to the visualization of the HIFU focus by using shockwave-generated bubbles and a diagnostic B-mode scanner. Bubbles were generated and pushed by shock waves toward the HIFU beam, and were trapped in its pressure valleys. These trapped bubbles moved along the pressure valleys and thereby delineated the shape and size of the HIFU beam. The main and sidelobes of 1.1- and 3.5 MHz HIFU beams were clearly visible, and could be measured with a millimeter resolution. The combined foci could also be visualized by observing the generation of sustained inertial cavitation and enhanced scattering. The results of this study further demonstrate the possibility of reducing the inertial cavitation threshold by the local introduction of shock wave-generated bubbles, which might be useful when bubble generation and cavitation-related bioeffects are intended within a small region in vivo. (C) 2005 Acoustical Society of America. [DOI: 10.1121/1.1904283]
\end{abstract}

PACS numbers: 43.58.Vb, 43.25.Cb, 43.35.Ei [RR]

Pages: $3740-3749$

\section{INTRODUCTION}

The minimally invasive nature of high-intensity focused ultrasound (HIFU) has been a focus of several recent therapeutic applications, especially tumor ablation. ${ }^{1-5}$ The temperature of the target tissue at the focus of a HIFU transducer can increase to more than $65^{\circ} \mathrm{C}$ within seconds, thus causing protein denaturing and cell death. However, the success of HIFU tumor ablation largely depends on the knowledge of the focusing properties of the treatment system, making techniques for quantitatively measuring the focal position and size essential.

Typical tools for measuring the position and size of a HIFU transducer are hydrophone scanning and schlieren optical imaging. ${ }^{6}$ A computer-controlled scanning system with

\footnotetext{
${ }^{a)}$ For correspondence: Dr. Chih-Kuang Yeh, Department of Electrical Engineering, Yuan Ze University, 135 Yuan-Tung Rd., Chung-Li, 320 Taoyuan, Taiwan. Telephone: +886-3-463-8800, ext. 2438 (voice); +886-3-4639355 (fax); electronic mail: ckyeh@saturn.yzu.edu.tw
}

a needle or membrane hydrophone can accurately determine the position, size, and pressure amplitude of the main and sidelobes of a HIFU sound field. Although a very fine resolution can be achieved, the scanning process is time consuming and the alignment of the hydrophone to the HIFU transducer is critical to the accuracy of the measurements. Moreover, the hydrophone is fragile and quickly saturated at high pressures, and the calibration of a hydrophone in a multielement HIFU system becomes difficult due to the acoustic waves coming from several sources differing greatly in their incoming angles. Schlieren optic imaging provides an overview of the shape and location of a HIFU focus as well as sidelobes and secondary foci. However, the resolution is low and thus the obtained results are more qualitative than quantitative.

In this article we report a novel method for estimating the position and size of the focus of a 1.1 or $3.5 \mathrm{MHz}$ HIFU transducer. The shapes and sizes of the main and sidelobes can be determined in a reasonably short time and with a high resolution. Furthermore, the changes in shape and pressures 
TABLE I. Simulation parameters.

\begin{tabular}{lcc}
\hline \hline Frequency $(f)$ & $1.1 \mathrm{MHz}$ & $3.5 \mathrm{MHz}$ \\
Transducer radius & $32 \mathrm{~mm}$ & $17.5 \mathrm{~mm}$ \\
Geometric focus (data from the & $62.64 \mathrm{~mm}$ & $55 \mathrm{~mm}$ \\
manufacturer) & & \\
Attenuation & $0 \mathrm{~Np} / \mathrm{cm}$ & $0 \mathrm{~Np} / \mathrm{cm}$ \\
Sound speed $(c)$ & $1500 \mathrm{~m} / \mathrm{s}$ & $1500 \mathrm{~m} / \mathrm{s}$ \\
Wavelength $(\lambda)$ & $1.364 \mathrm{~mm}$ & $0.429 \mathrm{~mm}$ \\
Grid size & & $0.1 \mathrm{~mm}$ \\
Field & $0.1 \mathrm{~mm}$ & $0.164 \mathrm{~mm}$ \\
Transducer $(\Delta x$ and $\Delta y)$ & $0.164 \mathrm{~mm}$ & \\
\hline \hline
\end{tabular}

where the foci of the two transducers intersect are also determined.

\section{EXPERIMENTAL MATERIALS AND METHODS}

\section{A. Experimental setup}

The experiments were performed in an acrylic tank (23 $\mathrm{cm} \mathrm{L} \times 15 \mathrm{~cm} \mathrm{~W} \times 15 \mathrm{~cm} \mathrm{H})$ filled with tap water. The experimental system consisted of four transducers (1.1 and 3.5 MHz HIFU transducers; H-101 and SU-102, Sonic Concepts, Woodinville, USA), a shock wave transducer (Piezoson 100 with a FB7 G2 probe, Richard Wolf, Knittlingen, Germany) for producing bubbles, and a diagnostic B-mode scanner (Titan with a L38 probe, Sonosite, Bothell, USA) for imaging. The L38 is a $5-10 \mathrm{MHz}$ broadband transducer with a central frequency of $7.5 \mathrm{MHz}$. The focus of the probe used with the shock wave generator was $1.7 \mathrm{~mm}$ in diameter and $6.7 \mathrm{~mm}$ in length, while the focal length was $20 \mathrm{~mm}$ from the edge plane of the probe (data from the manufacturer). The diameters and focal lengths of the 1.1 and $3.5 \mathrm{MHz}$ transducers are listed in Table I. The 1.1 MHz HIFU and the shock wave transducers were positioned orthogonally on two adjacent walls of the water tank, as shown in Fig. 1. For experiments on the combined beam pattern of two HIFU transducers, the second HIFU transducer $(3.5 \mathrm{MHz})$ was mounted on the wall

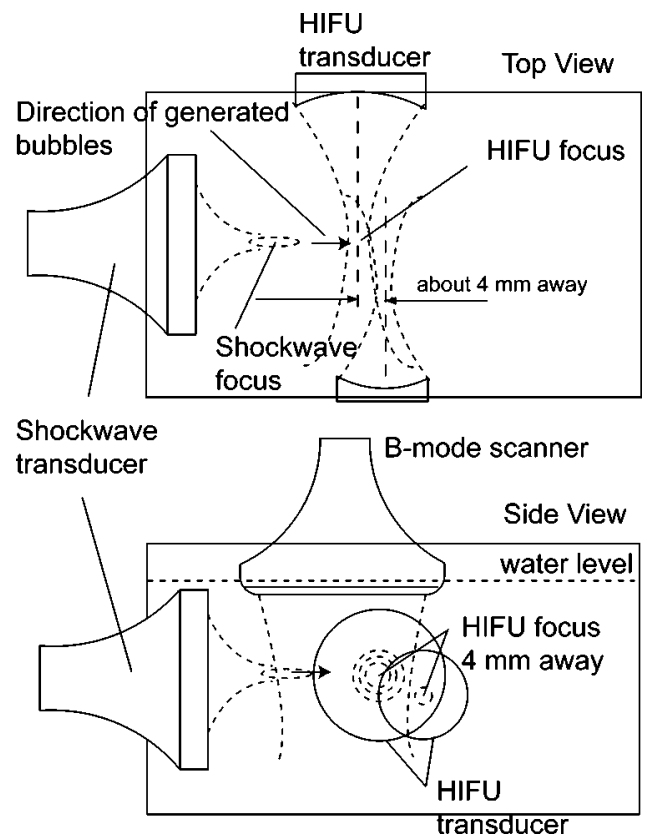

FIG. 1. Setup of the experimental apparatus: top and side views. facing the 1.1 MHz HIFU transducer with their central axes parallel to each other but separated by about $4 \mathrm{~mm}$. The B-mode probe scanned from the top of the water tank parallel to the axial direction of the shock wave transducer, i.e., cutting through the HIFU beam. The HIFU transducers were both mounted on 3-D positioners that allowed precise movement control.

The pressure values of the HIFU transducers were calculated from hydrophone (Onda, Sunnyvale, USA) pressure measurements performed in water at low output amplitudes that were linearly extrapolated to higher outputs. The peak negative pressures at the focus of the $1.1 \mathrm{MHz}$ transducer were 1.62 and $5.88 \mathrm{MPa}$ for outputs of 100 and $400 \mathrm{mV}$ from the function generator, respectively. The peak negative pressure of the $3.5 \mathrm{MHz}$ transducer was around $3 \mathrm{MPa}$ at the focal point. Two arbitrary function generators (33120A, Agilent, Palo Alto, USA; and DS345, Stanford Research Systems, Sunnyvale, USA) and two power amplifiers (150A250B and 150A100B, Amplifier Research, Souderton, USA) were used to drive the 3.5 and $1.1 \mathrm{MHz}$ HIFU transducers, respectively. The pulse repetition frequency (PRF) was $500 \mathrm{~Hz}$, and the pulse length was 100 cycles for both transducers. A clinical shock wave generator was used to induce inertial cavitation and generate bubbles, and was set to its highest output level (20, the corresponding peak negative pressure provided by the manufacturer was $19.0 \mathrm{MPa}$ ) and the highest PRF (4 Hz) for maximal bubble production. The beam-pattern images obtained by the diagnostic B-mode scanner were recorded on a VCR and off-line processed frame-by-frame using commercial software (Premiere Pro 1.5, Adobe Systems, San Jose, USA; Matlab, The MathWorks, Natick, USA).

\section{B. Experimental procedures}

\section{Series 1. Beam pattern of a single HIFU transducer}

In order to test the feasibility of using shock wavegenerated bubbles to visualize the beam pattern of HIFU transducers, the 1.1 and $3.5 \mathrm{MHz}$ transducers were first tested individually. The $1.1 \mathrm{MHz}$ HIFU transducer was driven at either 100 or $400 \mathrm{mV}$ and moved along its axial direction at a step size of 1 or $2 \mathrm{~mm}$, respectively. The shock wave generator produced and pushed bubbles toward the HIFU beam while B-mode images were taken continuously. After finishing "slicing" along the HIFU beam, the B-mode imaging plane was aligned with the HIFU focal plane again, and B-mode and color Doppler images were both taken. The output of the 1.1 MHz HIFU transducer was increased until enhanced scattering due to inertial cavitation at the center of the HIFU beam was induced. The beam pattern of the 3.5 $\mathrm{MHz}$ transducer was determined in a similar way.

\section{Series 2. Simulation}

To understand the beam profiles obtained in the above experiments, simulations were performed by modeling the 1.1 MHz HIFU transducer as a collection of point sources in a grid. For any point on the destination plane (fixed $z$ value), 
the following Rayleigh-Sommerfold integral ${ }^{7}$ was used to calculate the relative pressure amplitude contributed by each grid point of the source transducer:

$$
p(x, y, z)=i \frac{\rho c k}{2 \pi} \int_{S} \frac{u e^{-i k\left(r-r^{\prime}\right)}}{r-r^{\prime}} d S,
$$

where $i=\sqrt{-1}, \rho=$ tissue density, $c=$ sound speed, $k=$ wave number $(k=2 \pi / \lambda, \lambda$ is the wavelength, and $c=f \lambda$, where $f$ is sound frequency), $u=$ complex surface velocity of source, and $r-r^{\prime}=$ distance between a certain point on the transducer surface to a certain point in the acoustic field. For more efficient and convenient calculations, the above equation was simplified to

$$
p(x, y, z)=i \operatorname{AMP} \frac{\Delta x \Delta y}{2 \pi} \sum \sum \frac{e^{-i k\left(r-r^{\prime}\right)}}{r-r^{\prime}} .
$$

The amplitude of the simulated pressure field is relative when AMP is set to 1 , and $\Delta x$ and $\Delta y$ are the step sizes in the $x$ and $y$ directions. The parameters used in the simulation program are listed in Table I. The simulated results were then compared with the experimental results (see Sec. III).

\section{Series 3. Combined beam pattern of two HIFU transducers}

The major challenge in beam-pattern plotting is encountered during the testing of a multielement transducer (especially when the elements are at large angles to each other). To demonstrate the advantages of our newly developed method, two HIFU transducers (one 1.1 and the other 3.5 $\mathrm{MHz}$ ) were arranged facing each other, but with a $4 \mathrm{~mm}$ distance between their parallel central axes on purpose. The 3.5 MHz transducer was turned on first since its focal pattern was small and difficult to determine. The obtained B-mode images of the overlapping beam pattern were recorded and analyzed offline.

\section{RESULTS}

\section{A. Beam pattern of a single HIFU transducer}

To rapidly determine the focal size and location of a HIFU beam, the HIFU transducer was moved along its axial direction while B-mode images were taken sequentially. Figure 2(a) shows one of the recorded images near the focus of a $1.1 \mathrm{MHz}$ HIFU beam. When bubbles produced by the shock wave transducer were pushed toward the HIFU beam, a few concentric rings (white rings) appeared where bubbles were trapped and moved along the rings, which clearly visualized the locations of the pressure valleys around the HIFU focus (Fig. 3). The diameter of the central dark region was smaller for the $3.5 \mathrm{MHz}$ transducer than for the $1.1 \mathrm{MHz}$ transducer (Fig. 4). The radius of the first ring, which can be easily measured, was the radius of the first zero of the acoustic beam, and thus was larger than the focal radius (usually defined as being $6 \mathrm{~dB}$ below the maximum pressure) of the HIFU beam. In other words, the focal radius should be smaller than the diameter of measured radius of the first ring, $1.6 \mathrm{~mm}$ in Fig. 5(a). The number of visible rings increased with the pressure level [Fig. 2(b) and Fig. 5], whereas the diameter of the first ring was the same for low [Fig. 5(a)] and

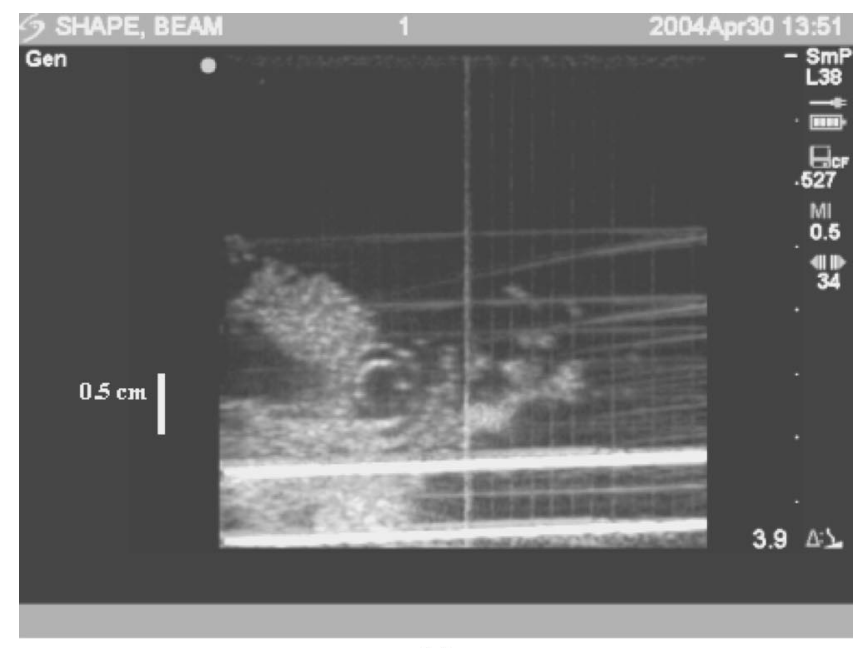

(a)

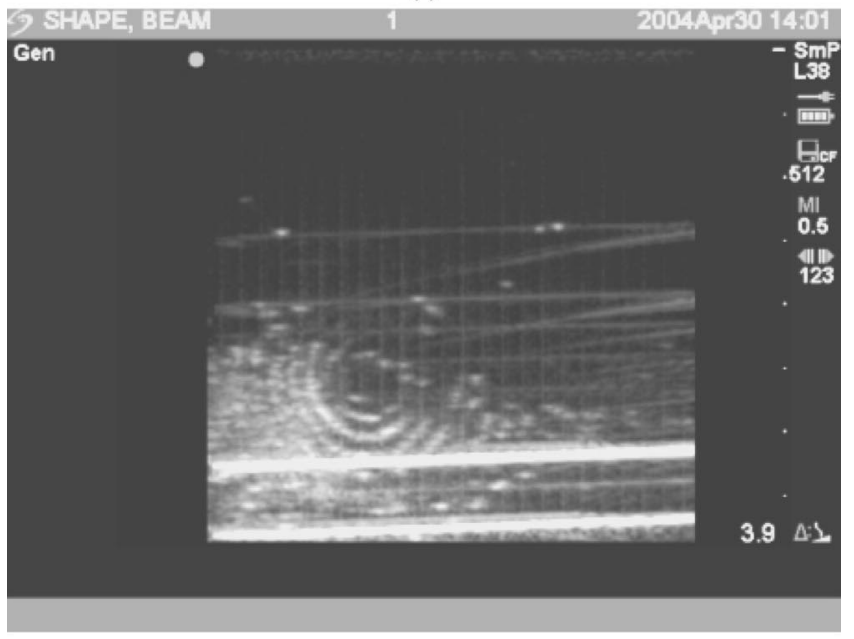

(b)

FIG. 2. (a) A B-mode image of the beam pattern of a $1.1 \mathrm{MHz}$ HIFU transducer near its focus. The output from the function generator is $100 \mathrm{mV}$ (producing a peak negative pressure of $1.62 \mathrm{MPa}$ ). The vertical white line was produced by a shock wave pulse. (b) A B-mode image of the beam pattern of a 1.1 MHz HIFU transducer at the same position as (a), but for a higher voltage output from the function generator $(400 \mathrm{mV}$, $5.88 \mathrm{MPa})$. More rings are clearly evident.

high [Fig. 5(b)] pressures. The minimum diameter of the central dark region occurred $48 \mathrm{~mm}$ from the edge plane of the 1.1 MHz HIFU transducer [Figs. 5(a) and 5(b)]. At a low-pressure condition, "radius steps" were seen at a few positions, e.g., 40-41, 42-45, 46-50, 50-54, and 55-59 $\mathrm{mm}$ [Fig. 5(a)]. At the transition from one step to the next one, the measured radii could be either at the upper "step" (case 2) or the lower "step" (case 1). The radius of the second ring (white triangle) for case 1 coincided with that of the first ring for case 2 (gray square).

Bubbles for visualizing the beam pattern of a HIFU transducer can be provided by means other than a shock wave transducer. Figure 6 shows the bubbles generated at the center of the HIFU focus by inertial cavitation at higher pressure levels (frame 3) that visualize the first and second rings of the HIFU beam when they moved outward. However, compared to the bubbles generated by a shock wave trans- 

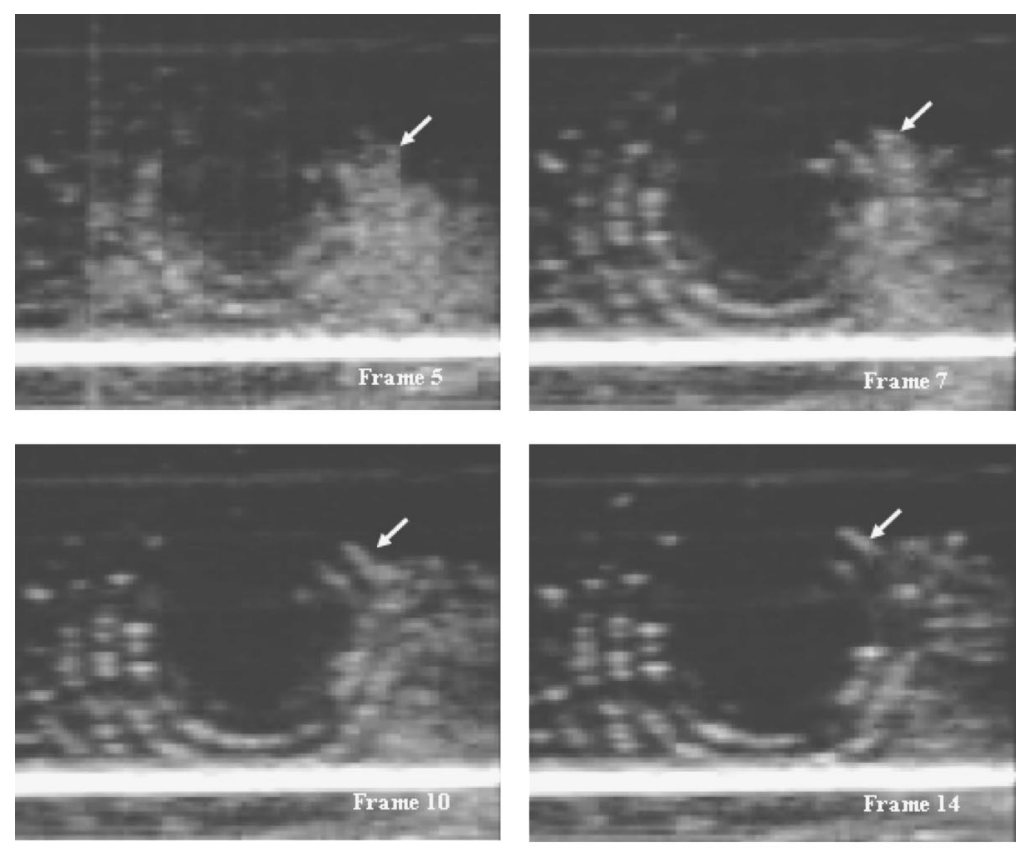

FIG. 3. A group of bubbles (white solid arrow) is moving along the third pressure valley (the third ring) of the 1.1 MHz HIFU beam. The acoustic parameters used in this test was $100 \mathrm{mV}$ output from the function generator, pulse length $=100$ cycles, and $P R F=500 \mathrm{~Hz}$. The B-mode imaging slice cut through the focal plane of the HIFU transducer (48 $\mathrm{mm}$ from the transducer's edge plane). In this figure, only frames $5,7,10,14,18$, and 26 are shown for simplicity.
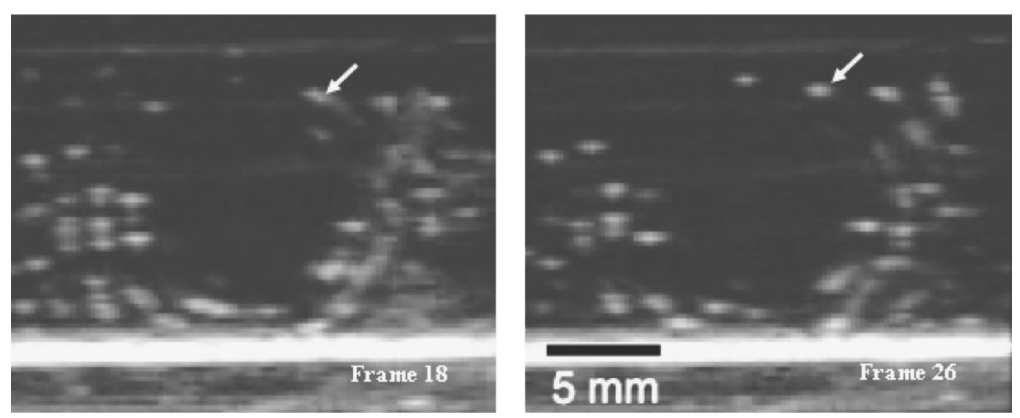

ducer, bubbles from the HIFU focus lasted for a shorter time and thus were probably smaller. Figure 7 shows a Doppler image of the bubbles generated by a HIFU transducer. An oscillating ball with rapidly changing color was evident at the focus of the HIFU transducer, which is indicative of the fast phase change in backscattered signals due to the generation and collapse of bubbles by inertial cavitation. This phenomenon was sometimes called the pseudo-Doppler shift and has been used to produce images of "stimulated acoustic emission."

\section{B. Matching experimental and simulated results}

In order to elucidate the physical meaning of the experimental results, the beam patterns of both 1.1 and $3.5 \mathrm{MHz}$ HIFU transducers were simulated and compared to the rings evident on the B-mode images. In Fig. 8, it is clear that the white rings of the $1.1 \mathrm{MHz}$ HIFU beam matched well with the location of the pressure valleys measured using a needle hydrophone. It is worth noting that the pressures at the valleys were not zero. The measured pressure might be the sum
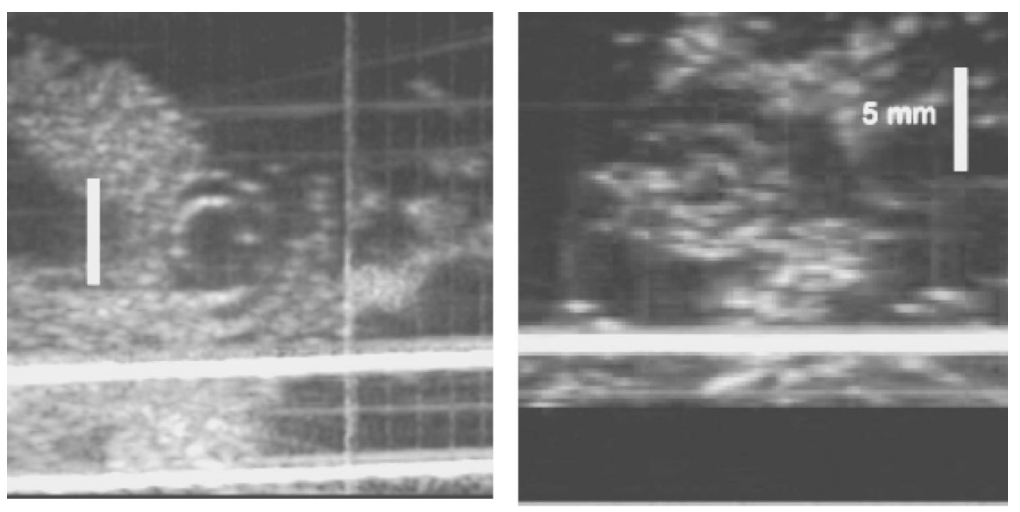

FIG. 4. The focal beam patterns of (a) $1.1 \mathrm{MHz}$ and (b) 3.5 MHz HIFU transducers. The B-mode imaging slice cut through the focal planes of both HIFU transducers. Comparing (a) and (b), the focal beam size of the 3.5 $\mathrm{MHz}$ transducer is smaller than that of the $1.1 \mathrm{MHz}$ one.

(a)

(b) 


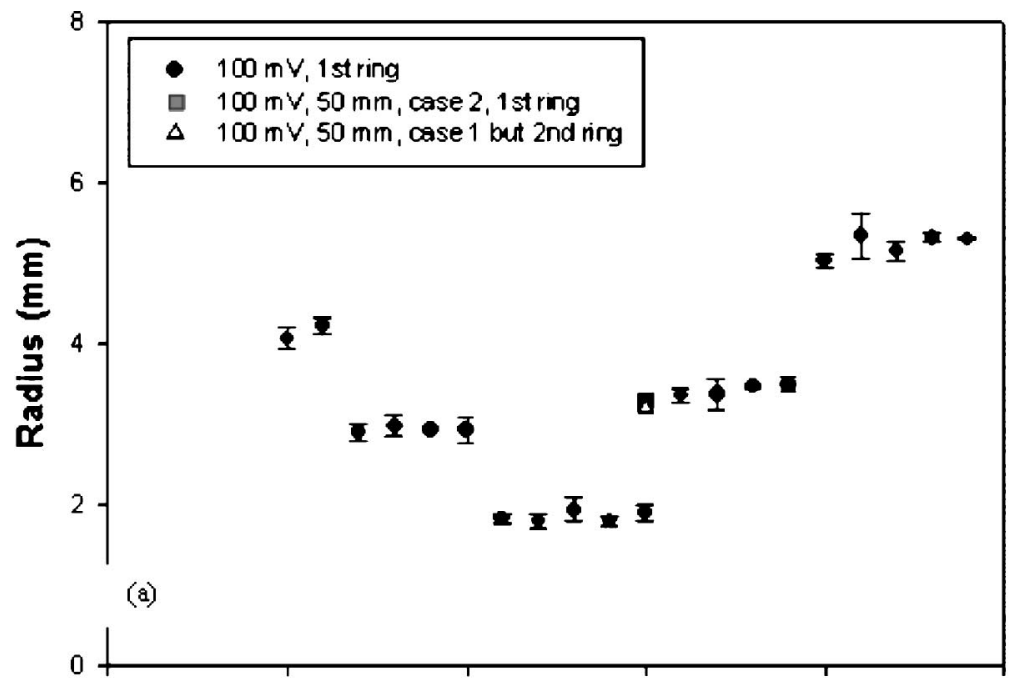

FIG. 5. The radii of each ring at (a) low- $(100 \mathrm{mV})$ and (b) high-pressure $(400 \mathrm{mV})$ output are shown. The minimum diameter of the $1.1 \mathrm{MHz}$ HIFU transducer occurred $48 \mathrm{~mm}$ from the edge plane. At a low-pressure condition, "radius steps" were seen at a few positions, e.g., from 46 to $50 \mathrm{~mm}$ and from 50 to $54 \mathrm{~mm}$. At the transition between two steps, e.g., at $50 \mathrm{~mm}$, the measured radii could be either at the upper "step" (case 2, gray square) or the lower "step" (case 1, black circle). The radius of the second ring (white triangle) for case 1 coincided with that of the first ring for case 2 (gray square). The error bar indicates the size of the standard deviation. At least 3 measurements were performed for each data point.

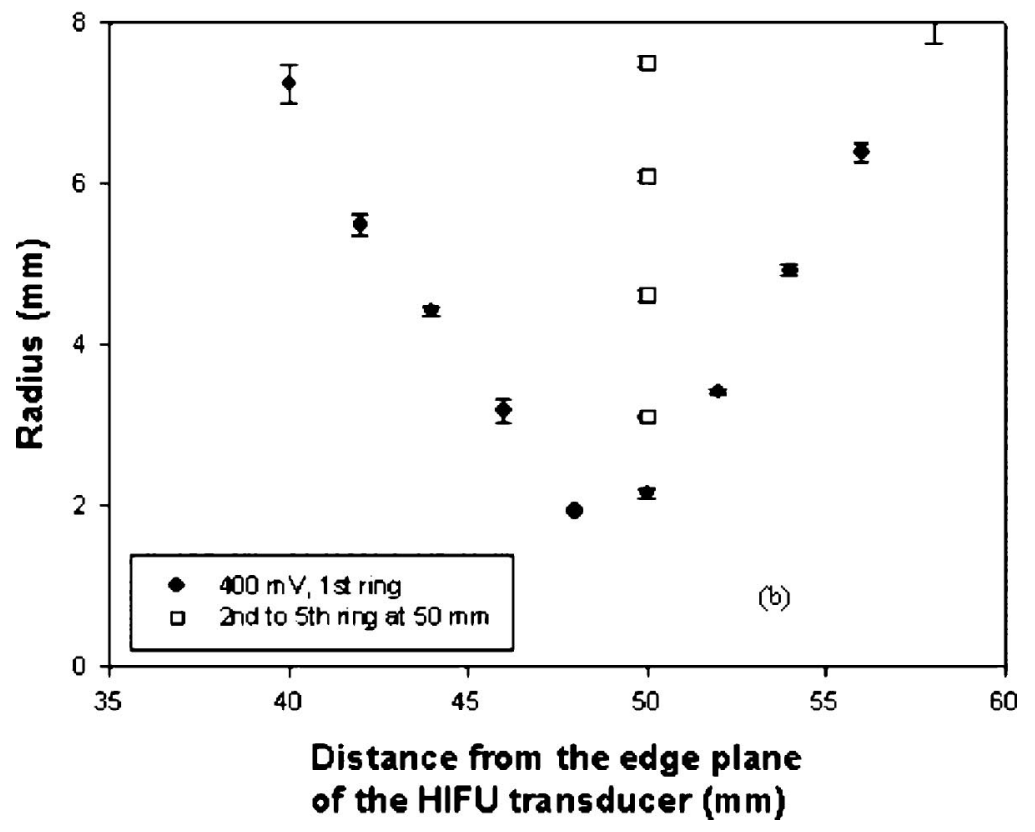

fore, before turning both transducers on, no bubble was generated near the confocal plane of both transducers after the bubbles from the shock waves passed or dissolved. When two transducers were turned on simultaneously, the pressures of both transducers summed up and thus the peak pressure level near the focus of the $3.5 \mathrm{MHz}$ transducer increased. The bubbles from the shock wave transducer probably acted as seeds of inertial cavitation and induced bubble generation. Continuous bubble generation was seen at the intersecting area and could persist up to a few minutes when bubbles from the shock wave generator disappeared for a long time. The enhanced scattering signals of the generated bubbles then clearly demonstrated the location of the intersecting area (Fig. 10).

To understand the beam pattern of two facing transducers, the focal location of each transducer was determined first. The focus of the $1.1 \mathrm{MHz}$ transducer was then moved by adjusting the 3-D positioner to make the focal planes of both the 1.1 and $3.5 \mathrm{MHz}$ beams coincide. The two foci were separated by $4 \mathrm{~mm}$ distance on purpose.

The pressure levels of the 1.1 and $3.5 \mathrm{MHz}$ HIFU transducers were both below the pressure threshold to induce inertial cavitation of bubbles provided by shock waves. There-

\section{DISCUSSION}

In this report we describe the results of a simple and novel method to determine the size and location of HIFU beams. The new method is particularly useful when analyzing a transducer array with a complex arrangement, for example, as used in the noninvasive ablation of a brain tumor 

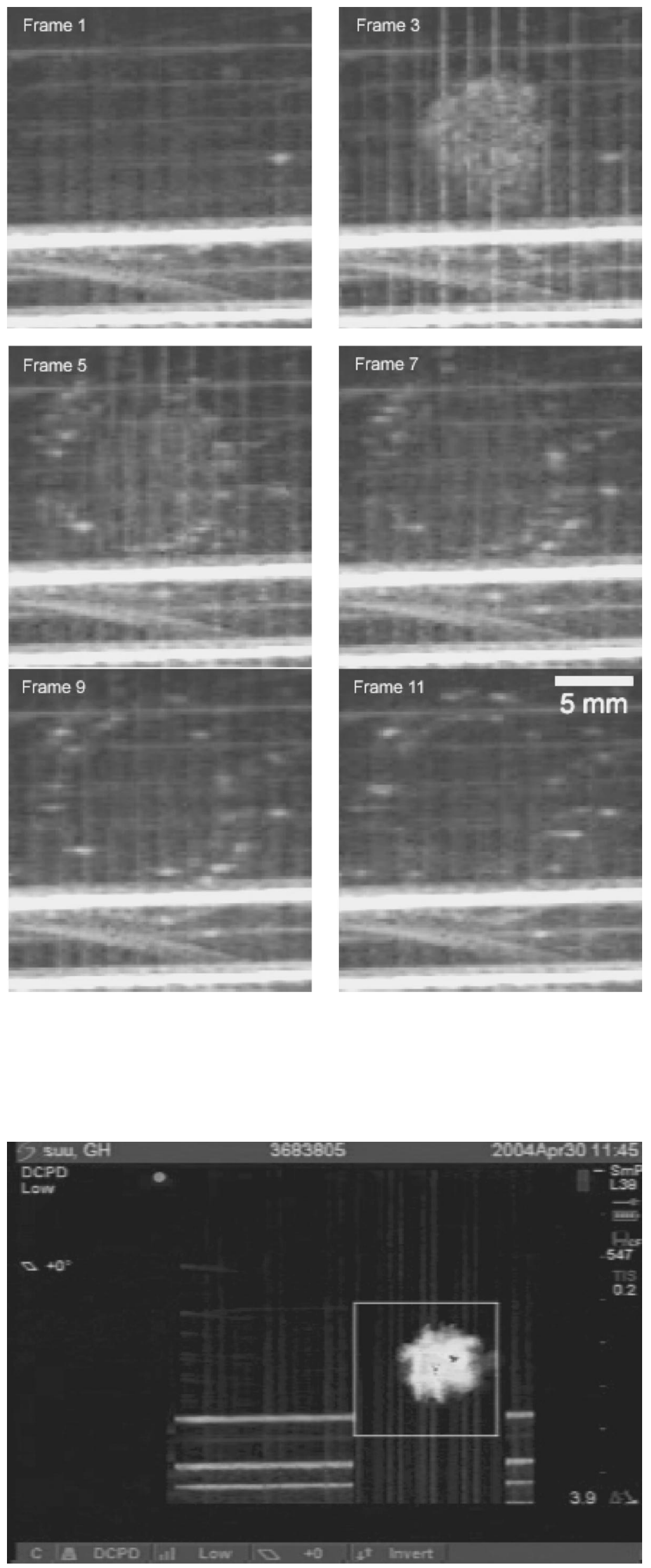

FIG. 6. The beam pattern of a $1.1 \mathrm{MHz}$ HIFU transducer was visualized by bubbles generated by inertial cavitation (frame 3 ). Two rings are clearly evident in frames 5-11 (550 mV or $\mathrm{P}=8.09 \mathrm{MPa}, 100$ cycles, 500 $\mathrm{Hz} \mathrm{PRF})$. The vertical white lines in each frame were produced by the HIFU transducer itself. Only frames 1, $3,5,7,9$, and 11 are shown.
FIG. 7. The bubbles generated by inertial cavitation at the focus of a $1.1 \mathrm{MHz}$ HIFU transducer looks like a ball changing in color rapidly (mosaic pattern) in a directional color power Doppler image $(550 \mathrm{mV}, 100$ cycles, $500 \mathrm{~Hz}$ PRF). 

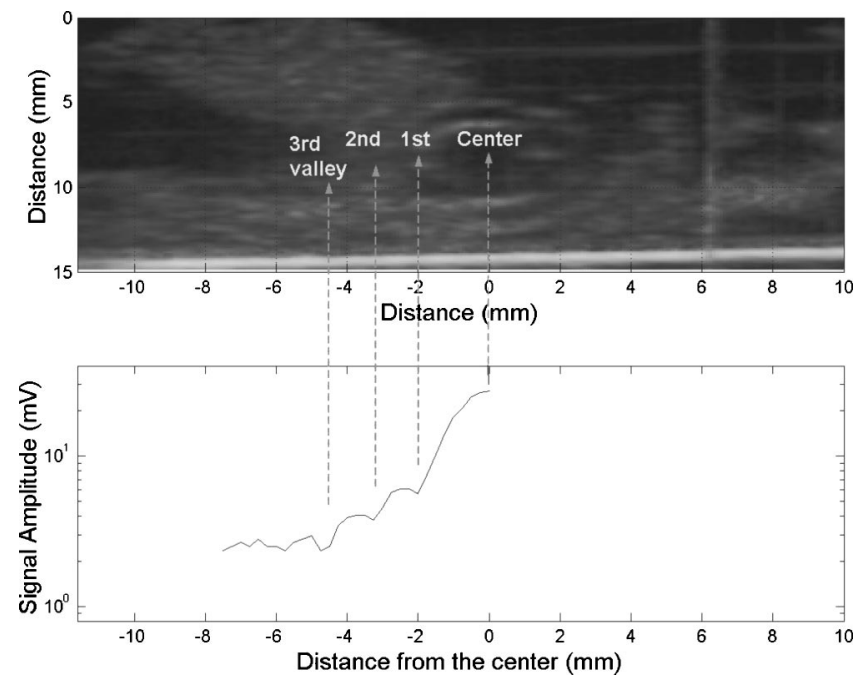

FIG. 8. The white rings of the $1.1 \mathrm{MHz}$ HIFU beam compared with the location of the pressure valleys measured by a needle hydrophone.

using a transcranial ultrasound array. Arranging two transducers from the bilateral temporal area of the skull-where the bone is thinner-may be preferable because the output from each transducer decreased and the possibility of overheating the brain tissue outside the target reduced. Unfortu- nately, the resulting beam pattern cannot be measured by a traditional needle hydrophone system. Furthermore, our proposed method can also be used to rapidly construct a 3-D beam pattern, in contrast to 3-D measurements with a needle hydrophone system usually taking many hours to complete. The proposed method is able to perform beam plotting of two facing transducers, and can construct a 3-D beam pattern in a relatively shorter time. However, the proposed method is not able to determine the absolute peak pressure or intensity, or the pressure profile of the focus. In addition, the resolution is limited by the frequency of the diagnostic ultrasound probe (in our case, a $7.5 \mathrm{MHz}$ central frequency), and thus is not adequate for a focal size smaller than $1 \mathrm{~mm}$. To clearly show a whole ring (the first pressure valley) in the B-mode images, an area of about $1 \times 1 \mathrm{~mm}^{2}$ (or about $5 \times 5$ pixels) is necessary. The use of a diagnostic probe with a higher frequency would increase the resolution.

Before turning on both transducers, the output of each transducer was lower than the inertial cavitation threshold of the bubbles from the shock wave generator, and hence no enhanced scattering was seen. When the foci of two transducers intersected, the peak pressure increased and exceeded these bubbles' inertial cavitation threshold. Therefore, before introducing bubbles to the intersecting area, no inertial cavitation or enhanced scattering was detected. Seeding some

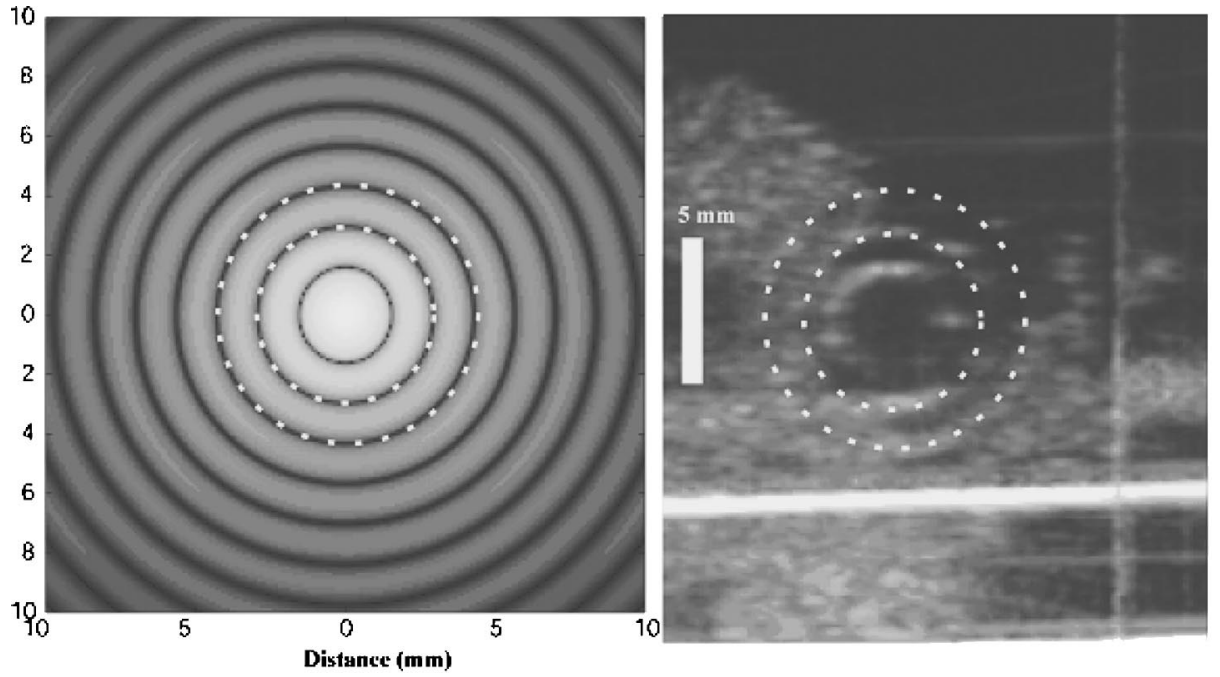

(a)

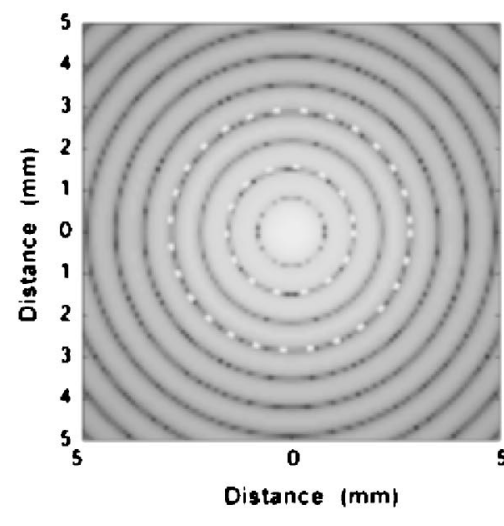

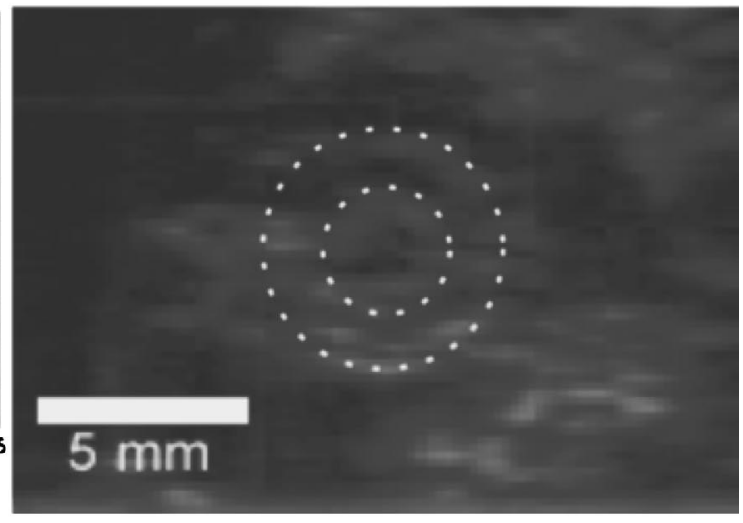

FIG. 9. The comparisons between the simulation and experimental results are shown for (a) the $1.1 \mathrm{MHz}$ and (b) the $3.5 \mathrm{MHz}$ HIFU transducers. For both transducers, the B-mode imaging planes were set at their focal planes. The scale of the simulation and experimental results are the same. For (a), dashed circles indicate the locations of the second and third pressure valleys for both simulation and experimental results. For (b), white dashed circles indicate the second and fourth pressure valleys.

(b) 

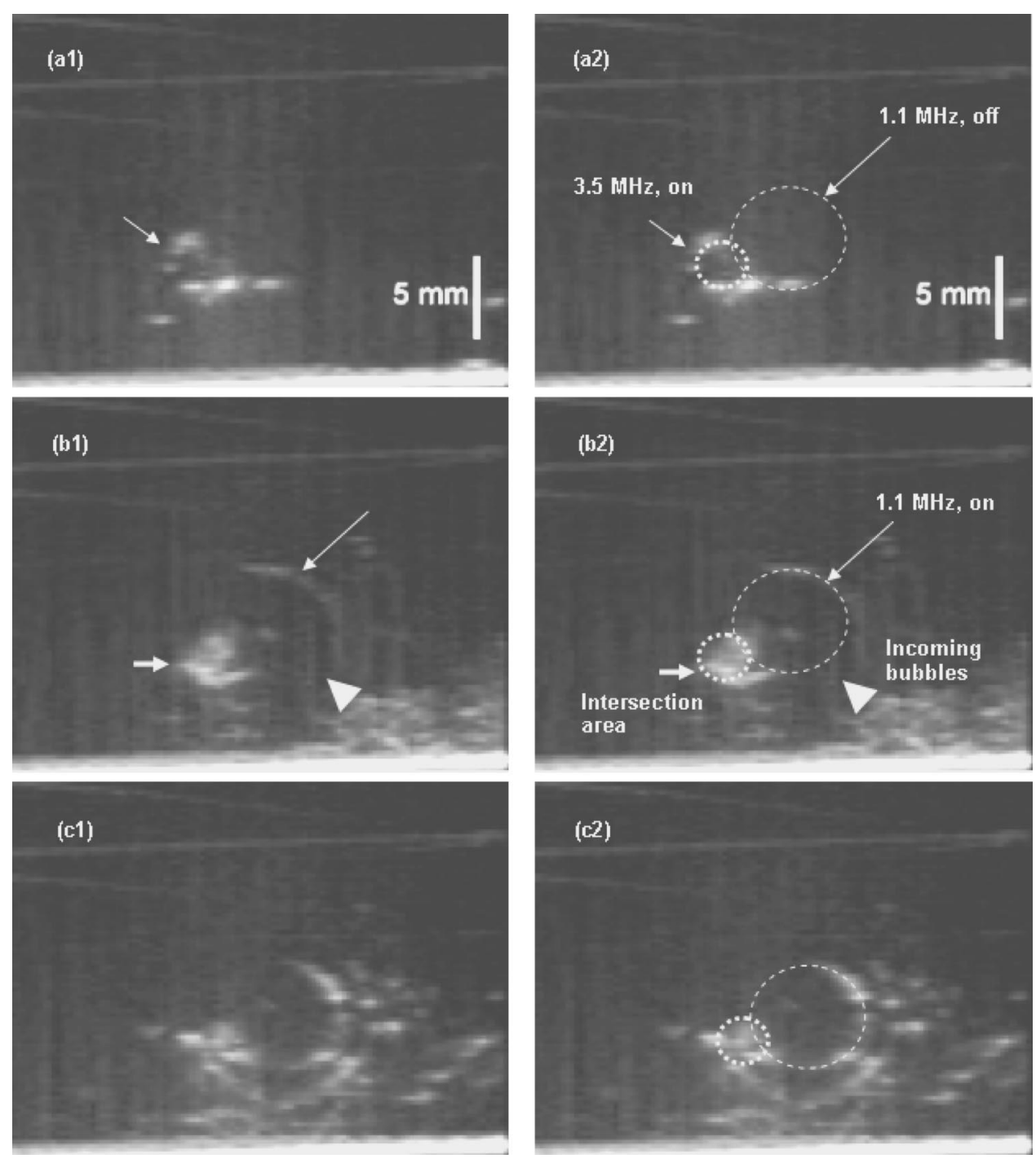

FIG. 10. The combined effect of the 1.1 and $3.5 \mathrm{MHz}$ HIFU transducers is shown. The left column (a1, b1, and c1) is the original B-mode images taken at the confocal plane of the two transducers. Locations of the first rings of both transducers are shown by thick (3.5 MHz) and thin (1.1 MHz) dashed rings. When the $3.5 \mathrm{MHz}$ HIFU transducer is on but the 1.1 one is off, the first ring of its beam pattern is clearly seen (thin arrow in a1 and a2). After turning on the $1.1 \mathrm{MHz}$ transducer and supplying the focal area with shock wave-generated bubbles, the focus of the $3.5 \mathrm{MHz}$ transducer filled with high-scattering signals, most likely bubbles (thick arrow in b1 and b2). The bubbles were generated and destroyed repeatedly at the focus of the 3.5 $\mathrm{MHz}$ beam. The moving direction of the shock wave-generated bubbles is indicated by the arrow heads (b1 and b2). The bubble-like signals at the focus of the $3.5 \mathrm{MHz}$ transducer can sustain for up to a few minutes after supplying "seed" bubbles generated by shock waves once. The $\mathrm{c} 1$ and $\mathrm{c} 2$ plots were taken after about a minute after stopping supplying bubbles from shock waves. bubbles by the shock wave transducer to the combined focus induced inertial cavitation, and the enhanced scattering was sustained for longer periods of time (up to a few minutes). In our case, the focal pressure of the $3.5 \mathrm{MHz}$ transducer increased by summation with the pressure of the first sidelobe of the $1.1 \mathrm{MHz}$ transducer, and exceeded the inertial cavitation threshold at $3.5 \mathrm{MHz}$. We believe that bubbles were generated, grew, and collapsed continuously at the intersecting area.

The schlieren imaging uses light to form images, which will not alter the sound field of the target transducer, and is pretty fast. However, the shlieren imaging method is basically a semiquantitative measurement of the pressure profile of the ultrasound beam. The bright and dark patterns projected on a screen can be used to determine the relative positions of the focus and sidelobes. Moreover, an absolute value of the beam size cannot be easily obtained. Furthermore, the schlieren imaging could be distorted easily at the presence of bubbles from inertial cavitation. The laser light diffracted while passing through moving bubbles and thus reduced the quality of the generated images. The new imaging method using bubbles can be used to perform measurement quantitatively. The obtained image quality even improves at the presence of bubbles from inertial cavitation.
The "step" behavior seen in Fig. 5 represented the true pressure profile around the HIFU focus. As seen in Fig. 11(a), the bright parts represent the pressure crests (peaks and saddles) while the dark parts are the pressure valleys. This background beam plot was created using the simulation equations described above. Bubbles tended to move to the low-pressure area and thus accumulated in the dark regions. The measured radii of the beam profile represented the inner most pressure valleys which bubbles could reach at different distances from the transducer surface. When the transducer's output was low, the pressure at the saddle part [A in Fig. 11(b)] was low enough to allow bubbles to climb over it and stayed in the first pressure valley, the lowest "step" (46-50 $\mathrm{mm}$ ). The pressure gradually increased while the distance to the transducer increased, and prevented bubbles from further climbing over. Bubbles stayed in the second pressure valley and formed a higher step (B, 50-54 mm). Ambiguity occurred at the transition between two steps. That is, bubbles can stay either in the lower or the higher pressure valley. Figure 11(b) shows the relative pressure amplitudes of the first pressure valley, the pressure crest, and the second pressure valley. However, when the output pressure was high enough [Fig. 5(b)], bubbles were more and more difficult to 


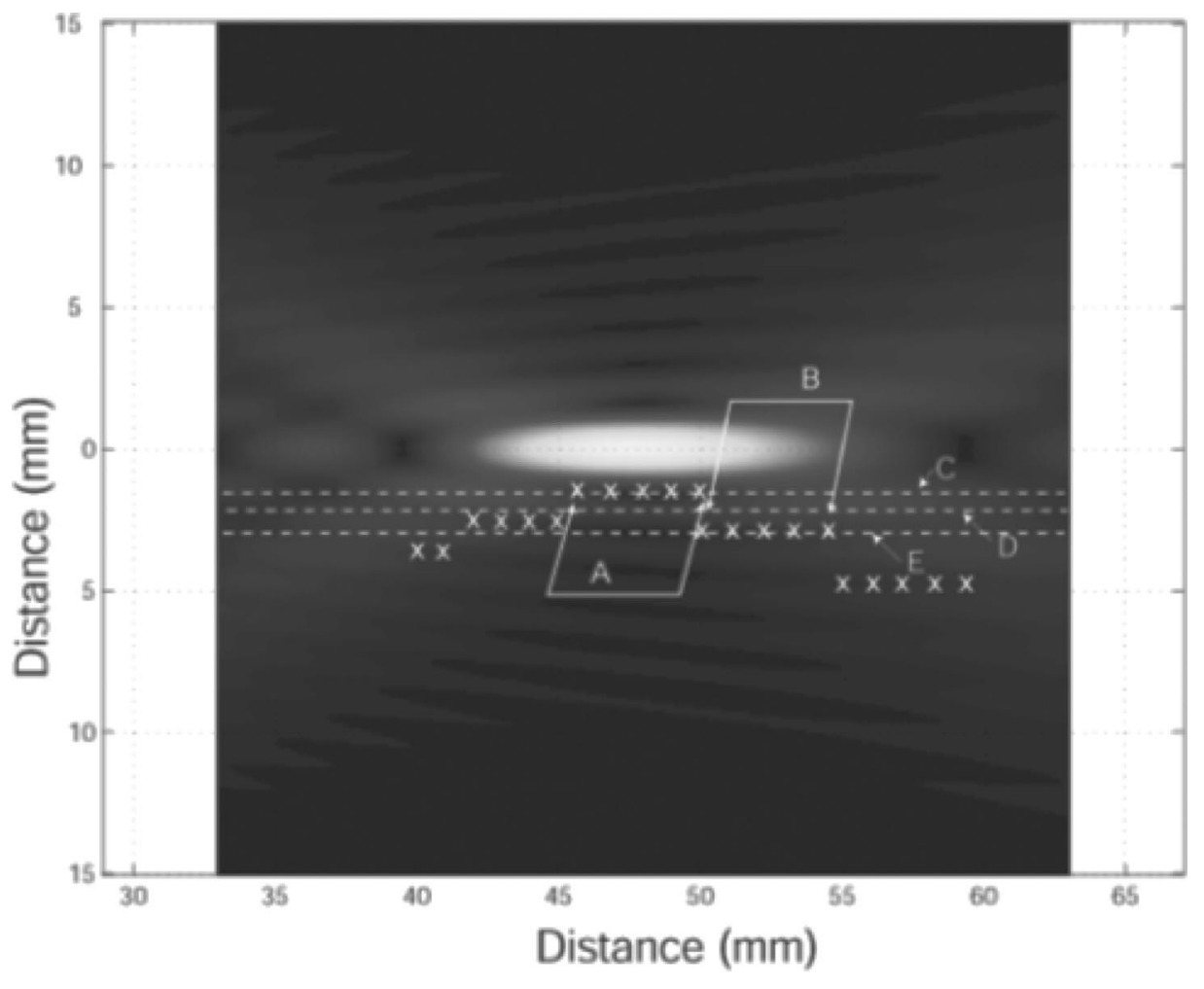

(a)

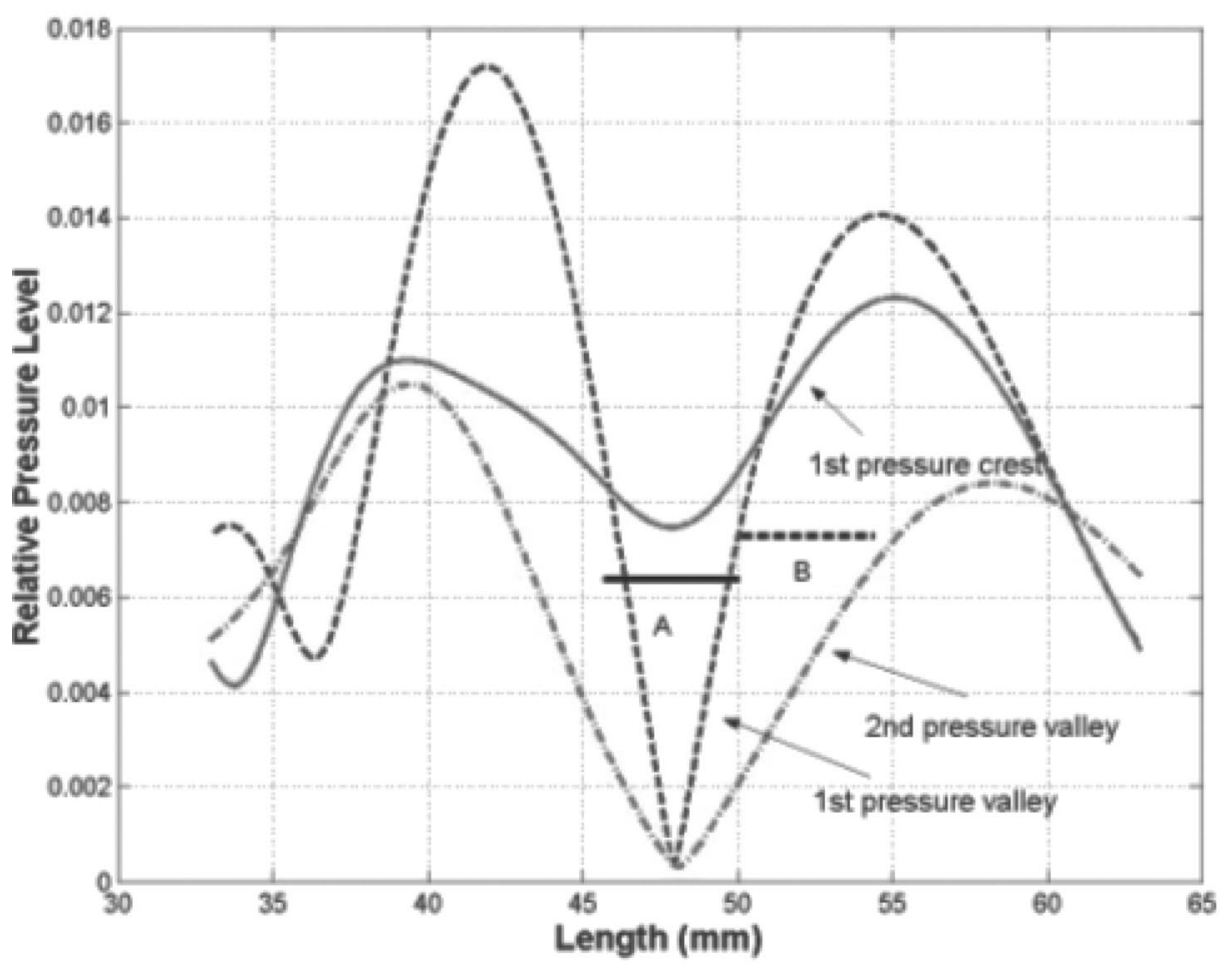

FIG. 11. (a) Simulated pressure profile of the $1.1 \mathrm{MHz}$ HIFU transducer near the transducer focus. " $\mathrm{X}$ " represents the positions where focal sizes were measured (see Fig. 5). "A" is the lowest pressure step at position 46 to 50 $\mathrm{mm}$. The pressure "crest (peak and saddle)" outside A is low and bubbles can climb over and enter "A" to form the first ring. " $\mathrm{B}$ " is where the second pressure "step" locates (50 to $54 \mathrm{~mm}$ ). The detailed pressure profiles of slice C (through step "A"), D (through first pressure crest), and E (through step "B") are shown in (b).

(b)

enter the inner pressure valleys and the measured radii thus increased rapidly.

Our observations indicate that shock waves can be used to provide nuclei to reduce the cavitation threshold and to locally induce inertial cavitation activity. The focal size of a shock wave is small $(1.7 \times 1.7 \times 6.7 \mathrm{~mm})$, and thus a large amount of energy can be directed to a small region in vivo.
The negative pressure of the shock wave was sufficiently low to induce cavitation and create bubbles in the intrahepatic vessels and bile. ${ }^{9,10}$ It is also possible for bubbles to be generated in loose tissue such as liver parenchyma. The shock wave-generated bubbles can be used to provide bubble seeds for further cavitation activity, and thus may be used to facilitate ultrasound-related bioeffects, such as drug delivery or 
gene transfection, when used with a therapeutic ultrasound transducer.

\section{ACKNOWLEDGMENTS}

This research was supported by grants from the National Science Council No. 92-2312-B-002-019-, and the National Taiwan University Hospital No. 93N011.

${ }^{1}$ S. Vaezy, V. Y. Fujimoto, C. Walker, R. W. Martin, E. Y. Chi, and L. A. Crum, "Treatment of uterine fibroid tumors in a nude mouse model using high-intensity focused ultrasound," Am. J. Obstet. Gynecol. 183, 6-11 (2000).

${ }^{2}$ G. ter Haar, D. Sinnett, and I. Rivens, "High intensity focused ultrasound-a surgical technique for the treatment of discrete liver tumours," Phys. Med. Biol. 34, 1743-1750 (1989).

${ }^{3}$ F. Wu, Z. B. Wang, W. Z. Chen, J. Z. Zou, J. Bai, H. Zhu, K. Q. Li, F. L. Xie, C. B. Jin, H. B. Su, and G. W. Gao, "High intensity focused ultrasound for extracorporeal treatment of solid carcinomas: four-year Chinese clinical experience," Proceedings of the International Symposium of Therapeutic Ultrasound, 2002, pp. 34-43.
${ }^{4}$ R. Yang, C. R. Reilly, F. J. Rescorla, P. R. Faught, N. T. Sanghvi, F. J. Fry, T. D. Franklin, Jr., L. Lumeng, and J. L. Grosfeld, "High-intensity focused ultrasound in the treatment of experimental liver cancer," Arch. Surg. (Chicago) 126, 1002-1009 (1991).

${ }^{5}$ J. Y. Chapelon, M. Ribault, F. Vernier, R. Souchon, and A. Gelet, “Treatment of localised prostate cancer with transrectal high intensity focused ultrasound," Eur. J. Ultrasound 9, 31-38 (1999).

${ }^{6}$ U. Schatzle, T. Reuner, J. Jenne, and A. Heilingbrunner, "Quality assurance tools for therapeutic ultrasound," Ultrasonics 36, 679-682 (1998).

${ }^{7}$ H. T. O'Nell, "Theory of focusing radiators," J. Acoust. Soc. Am. 21, 516-526 (1949).

${ }^{8}$ M. Blomley, T. Albrecht, D. Cosgrove, V. Jayaram, J. Butler-Barnes, and R. Eckersley, "Stimulated acoustic emission in liver parenchyma with Levovist," Lancet 351, 568 (1998).

${ }^{9}$ M. Delius, R. Denk, C. Berding, H. G. Liebich, M. Jordan, and W. Brendel, "Biological effects of shock waves: cavitation by shock waves in piglet liver," Ultrasound Med. Biol. 16, 467-472 (1990).

${ }^{10}$ L. E. Forer, W. J. Davros, J. Goldberg, F. al Kawas, B. S. Garra, W. Hayes, and R. K. Zeman, "Hepatic cavitation. A marker of transient hepatocellular injury during biliary lithotripsy,” Dig. Dis. Sci. 37, 1510-1516 (1992). 
Copyright of Journal of the Acoustical Society of America is the property of American Institute of Physics and its content may not be copied or emailed to multiple sites or posted to a listserv without the copyright holder's express written permission. However, users may print, download, or email articles for individual use. 